\title{
The prostate stem cell antigen represents a novel glioma-associated antigen
}

\author{
K.D. GEIGER ${ }^{1 *}$, S. HENDRUSCHK ${ }^{2}$, E.P. RIEBER ${ }^{3}$, A. MORGENROTH ${ }^{3}$, B. WEIGLE ${ }^{3}$, \\ T. JURATLI ${ }^{2}$, V. SENNER ${ }^{4}$, G. SCHACKERT ${ }^{2}$ and A. TEMME ${ }^{2 *}$ \\ ${ }^{1}$ Department of Neuropathology, Institute for Pathology, Medical Faculty Carl Gustav Carus; ${ }^{2}$ Department \\ of Neurosurgery, University Hospital Carl Gustav Carus; ${ }^{3}$ Institute of Immunology, Medical Faculty \\ Carl Gustav Carus, Technical University Dresden, D-01307 Dresden; ${ }^{4}$ Institute of \\ Neuropathology, University Hospital Muenster, D-48149 Muenster, Germany
}

Received January 21, 2011; Accepted March 16, 2011

DOI: $10.3892 /$ or.2011.1265

\begin{abstract}
Gliomas of WHO grades III-IV are malignant brain tumors mostly resistant to conventional therapies. Therefore, novel strategies for the treatment of gliomas are warranted. Although immunotherapy is gaining increased attention for the treatment of malignant gliomas and in particular of glioblastoma multiforme (GBM), this approach requires the identification of appropriate antigens. Our aim was to investigate the expression of the prostate stem cell antigen (PSCA), a highly $\mathrm{N}$-glycosylated phosphatidylinositol (GPI)-anchored cell surface protein, in gliomas of different WHO grades in order to evaluate its potential as a diagnostic marker and as a target for immunotherapy. Tumor specimens and controls were assessed by quantitative RT-PCR, Western blotting and immunohistochemistry. The samples investigated in the study consisted of 210 human glial tumors, among which 31 were oligodendrogliomas, 9 ependymomas and 170 were astrocytomas (including 134 glioblastomas). PSCA was absent in normal brain tissue, but was detected in WHO grade III-IV gliomas. Weak PSCA protein expression was also recognized in some WHO grade I and WHO grade II tumors. The difference between WHO grade I-II tumors and WHO grade III-IV tumors was statistically significant $(\mathrm{p}<0.001)$. Our results suggest that increased PSCA expression levels are
\end{abstract}

Correspondence to: Dr A. Temme, Department of Neurosurgery, Section Experimental Neurosurgery/Tumor Immunology, University Hospital Carl Gustav Carus, TU Dresden, Fetscherstrasse 74, D-01307 Dresden, Germany

E-mail: achim.temme@uniklinikum-dresden.de

*Contributed equally

Abbreviations: GBM, glioblastoma multiforme; PSCA, prostate stem cell antigen; QRT-PCR, quantitative real-time polymerase chain reaction; TAA, tumor-associated antigen

Key words: prostate stem cell antigen, tumor-associated antigen, gliomas, RT-PCR linked to gliomas of WHO grades III and IV, and may represent a suitable additional target for immunotherapy of gliomas.

\section{Introduction}

Glioblastoma multiforme (GBM) a glioma of WHO grade IV, accounts for $60-75 \%$ of all astrocytic tumors that due to its genetic instability gives rise to heterogeneous tumor cell descendants and possesses the capability of invading adjacent normal brain tissue (1). Despite improvements in neurosurgery, chemotherapy and radiotherapy, only a subset of patients suffering from GBM survives longer than 18 months (2).

The use of either antibody-based or T-cell-mediated immunotherapy to selectively kill remnant glioma cells that could not be completely removed by surgery because of the infiltration of the tumor into the adjacent brain tissue has received increasing attention. A number of animal models have shown that gliomas may be amenable to immune therapeutic approaches $(3,4)$. Moreover, the identification of humoral as well as cellular immune responses in brain tumor patients triggered efforts to activate the immune system against glioma cells $(5,6)$. Although, some tumor-associated antigens (TAA) including EGFRvIII (7), HER2-neu (8,9), gp100 (9), MAGE-1 (9), MART (10), hTERT $(10,11)$, survivin (12) and members of the SOX-family have been identified $(6,13-15)$ the extraordinary diversity of glioblastomas call for the identification of more TAAs to efficiently target the heterogeneous tumor cells (16).

Our focus has been the identification of surface TAAs which distinguish tumor cells from normal cells and which can be used for antibody-mediated immunotherapy and for diagnostic purposes. A likely candidate, the prostate stem cell antigen (PSCA) is related to the Ly-6/Thy-1 family of cell surface antigens that bears $30 \%$ homology to stem cell antigen type 2 (SCA-2) (17,18). It is an N-glycosylated phosphatidylinositol (GPI)-anchored cell surface protein and of particular interest since it is overexpressed in prostate carcinoma (PCa) (19) as well as in PCa metastases (20). Strong expression of PSCA has also been detected in bladder cancer (21), pancreatic adenocarcinoma (22) and clear cell carcinoma (23).

Interestingly, PSCA-antibodies and -reactive CD8-positive $\mathrm{T}$ cells have been found in the serum of patients suffering from 
pancreatic adenocarcinoma and PCa, respectively $(24,25)$. In addition, PSCA vaccination induced a long-term immune response against prostate cancer in transgenic adenocarcinoma mouse prostate (TRAMP) mice in the absence of autoimmunity (26). First experimental approaches to treat PCa using anti-PSCA antibodies also showed promising results which confirms PSCA as a suitable target for immunotherapeutic strategies $(27,28)$.

In order to identify novel surface antigens selectively expressed in malignant gliomas we screened GBM specimens for the expression of PSCA mRNA. We found that PSCA mRNA was expressed in $\sim 40 \%$ of the GBM samples, whereas PSCA expression in normal brain samples was negligible. Immunohistological analysis of GBM and additional analysis of WHO III brain tumors confirmed the PSCA expression in malignant gliomas. In addition, weak PSCA immunoreactivity (IR) was found in 12 out of 28 benign WHO grade I and II tumors. We suggest that for a significant number of patients with malignant gliomas, PSCA might represent a suitable target for an immunotherapeutic approach to eliminate glioma cells.

\section{Materials and methods}

cDNA synthesis and quantitative PCR. For quantitative PCR analysis of PSCA transcripts, cDNA synthesis was performed using $1 \mu \mathrm{g}$ total mRNA from normal brain or from glioblastoma specimens, oligo(dt)12-15 primers (GE Healthcare, Freiburg, Germany) and the Omniscript reverse transcriptase kit (Qiagen, Hilden, Germany) according to the manufacturer's protocol. The amount of specific PSCA-PCR product was determined by detection of the fluorescence signals of the intercalating dye SYBR-Green, using the LightCycler FastStart DNA master SYBR-Green I kit (Roche, Mannheim, Germany). The amplification of PSCA was performed according to Cunha et al (29). The PSCA mRNA cDNA copy number was calculated in relation to the amplification product amounts of internal standards and was normalized to the quantitative measurements of the housekeeping gene $\beta$-actin. The amount of $\beta$-actin was determined using the primers LC- $\beta$-actin forward, 5'-tcaccgagcgcggct-3' and reverse, 5'-taatgtcacgcattt ccc-3' applying an amplification protocol consisting of a denaturation step at $95^{\circ} \mathrm{C}$ for $10 \mathrm{~min}$, followed by 40 cycles with a $95^{\circ} \mathrm{C}$ denaturation step for $15 \mathrm{sec}, 61^{\circ} \mathrm{C}$ annealing for $5 \mathrm{sec}$ and a $72^{\circ} \mathrm{C}$ extension step for $5 \mathrm{sec}$. Quantity standard curves were generated applying dilutions over 6-log scales $\left(10^{1}-10^{6}\right)$ per capillary using a pREV-CMV-PSCA-IRES2-EGFP vector and an pEGFP-actin vector (Takara-Clontech, Heidelberg, Germany) containing the full coding region of PSCA and $\beta$-actin, respectively. GBM samples were rated positive when they showed a significant difference $(\mathrm{p}<0.05)$ to four light cycler runs using cDNAs of two normal brain samples (mean expression level $3 \pm 2$ copies PSCA mRNA/1000 copies actin), obtained from epilepsy neurosurgery, assessed by the Student's t-test. In order to compare QRT-PCR expression levels to immunohistochemistry results PSCA mRNA copy numbers from 10 to 39 copies/1000 copies actin mRNA were rated as moderate expression. Expression of PSCA $>40$ copies mRNA/1000 copies actin mRNA was rated as strong expression.
Cell culture. Fresh GBM tissue for research purposes was provided by the Department of Neurosurgery, University Hospital Carl Gustav Carus, and was approved by the ethics committee of the Medical Faculty Carl Gustav Carus, TU Dresden. The fresh tumor specimens were divided for routine histopathology, cryopreservation and cell culture. To obtain primary glioma cells a tumor fragment was minced in DMEM medium supplemented with $20 \%$ (v/v) fetal calf serum (PAA, Pasching, Austria), 4X non-essential amino acids (Gibco-BRL, Eggenstein, Germany) $100 \mathrm{U} / \mathrm{ml}$ penicillin and $0.1 \mathrm{mg} / \mathrm{ml}$ streptomycin (PAA). The cells were filtered through gauze and were centrifuged at $4^{\circ} \mathrm{C}$ and $600 \mathrm{x} \mathrm{g}$, for $10 \mathrm{~min}$. Cell pellets were dispersed in fresh medium and the cell viability was determined by trypan blue exclusion. Equal aliquots of cells were plated on poly-L-lysine coated culture flasks. Every 2 days the medium was replaced with fresh medium. Upon $60 \%$ confluency the cells were split and consecutively adapted to normal cell culture flasks.

Indirect immunofluorescence analysis and FACS analysis. Primary GBM cell preparations were grown on cover slides and fixed in ice-cold paraformaldehyde for $20 \mathrm{~min}$. After washing in phosphate-buffered saline (PBS), cells were permeabilized with $1 \%$ sodium citrate/ $0.1 \%$ Triton $\mathrm{X}-100$ and washed three times with PBS containing $0.1 \%$ bovine serum albumin (BSA). Cells were incubated for $1 \mathrm{~h}$ at room temperature with an affinity-purified monoclonal anti-PSCA preparation, described previously [clone 7F5, IgG1-isotype (30)]. After washing again with PBS/0.1 \% BSA, the cells were incubated for $1 \mathrm{~h}$ at room temperature with $\mathrm{Cy} 3$-conjugated anti-mouse IgG (stock solution, 1:50 diluted, as recommended by the supplier; Dianova, Hamburg, Germany). After intensive washing with PBS the DNA was counterstained with Hoechst 33342. Cells were examined by fluorescence microscopy (Olympus IX70, Hamburg, Germany). FACS analysis was performed using a FACScan and the Cell Quest software (Becton-Dickinson, Heidelberg, Germany) with non-fixed cells using the anti-PSCA clone 7F5 and secondary antimouse-IgG-Cy3 (Dianova). As an internal positive control, we used 293T cells transduced with a retroviral vector encoding full length PSCA linked with EGFP via internal ribosomal entry sites, designated as 293T-PSCA-IRES2-EGFP. As a negative control we used 293T wild-type cells. For the analysis of PSCA expression on 293T-PSCA-IRES2-EGFP and primary glioma cells an IgG1 isotype control antibody (Becton-Dickinson) was included.

Western blot analysis. For protein analysis of the PSCA expression liquid nitrogen-frozen tissues were homogenized using a mortar and pestle and total protein lysates were prepared in non-reducing 5X SDS sample buffer (15\% SDS, $50 \%$ glycerol, and $0.15 \%$ bromophenol blue). Protein samples were subjected to electrophoresis and blotted onto PVDF membranes (PALL, Dreieich, Germany). As positive control we used total protein lysates from 293T-PSCA-IRES2EGFP cells. PSCA immunostaining was performed with the monoclonal 7F5 antibody; tubulin was detected by the use of a monoclonal anti-tubulin antibody (Sigma, St. Louis, MO; clone DM 1A, dilution 1:500). The secondary goat anti-mouse antibody coupled to horseradish peroxidase (both 


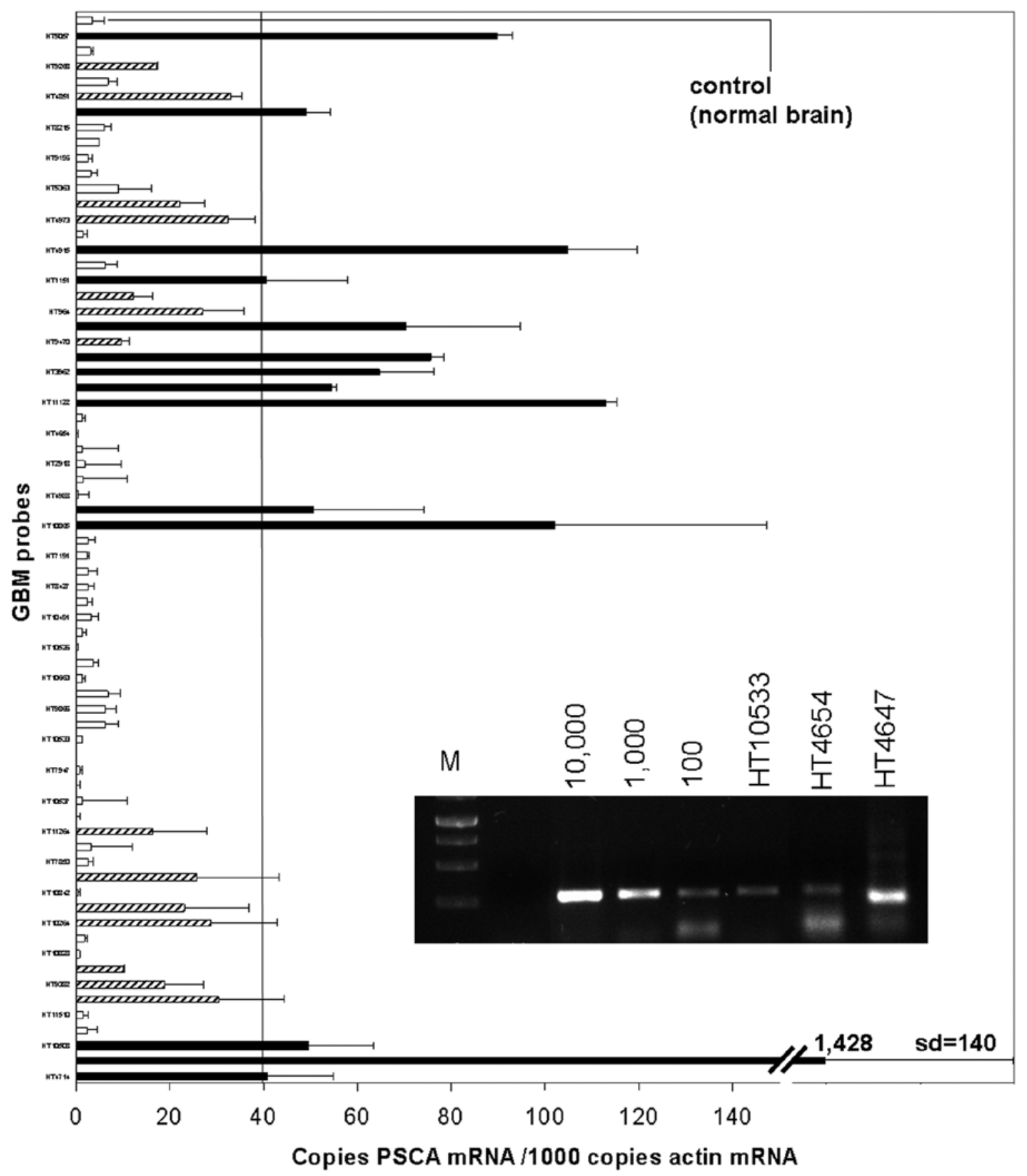

Figure 1. QRT-PCR analyses of PSCA-mRNA in GBM samples. GBM samples displaying up to 39 copies PSCA mRNA/1000 copies actin mRNA (moderate expression, striped columns), and displaying 40 and more copies PSCA mRNA/1000 copies actin mRNA (strong expression, black columns) are indicated. Samples showing no significant difference in expression compared to the mean of two QRT-PCR analyses of normal brain controls are depicted (white columns). Bars represent the standard derivation of the mean. Inlet shows a DNA agarose gel of representative light cycler probes and standard dilutions using a DNA-vector encoding PSCA as described in Materials and methods.

diluted 1:2500, Dako, Hamburg, Germany) was visualized with enhanced chemiluminescence (ECL Plus, Amersham Bioscience) as recommended by the supplier.

Immunohistochemistry and statistics. A total of 210 human glial tumors, confirmed by certified neuropathologists according to the WHO classification (1), and 10 normal control tissues from autopsy brains were tested for the expression of PSCA by immunohistochemistry (IHC/IR) using the indirect peroxidase technique on tissue multi-arrays (TMA) of paraffin-embedded specimens. The 210 glial tumors included 31 oligodendro- gliomas WHO grade II and III, 9 ependymonas WHO grade II, 4 astrocytomas WHO grade I, 12 astrocytomas WHO grade II, 20 astrocytomas WHO grade III, and 134 glioblastomas which consisted mostly of primary glioblastomas. In total, there were 28 benign gliomas of WHO grades I and II, and 182 malignant gliomas of WHO grades III and IV. Additionally, we included 5 cases with epilepsy due to hippocampal sclerosis and 10 cases with reactive astrocytosis due to HIV-encephalopathy using the indirect peroxidase technique on TMA containing 1-5 samples per case with a core size of $1.5 \mathrm{~mm}$ in diameter. Each TMA contained positive and negative controls. For the 
HIV cases whole sections of autopsy tissues were also used. Additionally, the results of TMA were compared to the whole sections of the same tumor sample from five cases. To detect PSCA by IHC, we used the monoclonal mouse antibody 7F5 and an indirect peroxidase system (LSAB-II, Dako) with diaminobenzidine (DAB, Sigma) as a chromogen on paraffin-embedded tissues. Staining of paraffin sections with an IgG1 isotype control antibody and with non-fat milk 5\% (from powder; Spinnrad, Lübeck, Germany) was performed to exclude non-specific staining by LSAB-II. Evaluation of immuno-staining on TMA was performed without knowledge of the diagnosis on 5- $\mu \mathrm{m}$ sections, at a magnification of $\times 20$ in the light microscope equipped with a graded ocular lens (10x 10 squares, corresponding to $500 \times 500 \mu \mathrm{m}$ at $\times 20$ magnification) (Carl Zeiss, Oberkochen, Germany). The immunoreactivity (IR) measurements were repeated at least twice and scored according to a semi-quantitative scoring system in comparison to normal controls. For statistical purposes only vital areas of the tumors were counted. Score values were distributed as follows: 0 , no positive cells (as in normal astrocytes); $1+$, weak staining in $<50 \%$ of the tumor cells; $2+$, moderate staining in $50-75 \%$ of tumor cells; $3+$, strong staining in $>75 \%$ of the tumor cells with mostly membranous staining or staining of astrocytic processes. For statistical analysis the univariate $\chi^{2}$ test and the corrected Pearson's contingency coefficient for non-parametric correlation was applied.

\section{Results}

PSCA expression in human glioblastoma samples. We examined 69 pathologically confirmed human GBM specimens for the presence of PSCA mRNA by QRT-PCR (Fig. 1). As a control we included specimens of normal brains obtained in the course of epilepsy neurosurgery. We noticed that approximately $40 \%$ of all tested GBM specimens were positive for PSCA mRNA expression. Interestingly, half of these probes were moderately positive for PSCA (up to 39 copies PSCA mRNA per 1000 molecules actin) and the other half of the positive probes showed a strong mRNA expression of PSCA ( 39 copies PSCA mRNA per 1000 molecules actin mRNA). In $60 \%$ of GBM probes no significant increase in PSCA levels was observed when compared to a normal brain sample, which was included as an internal control. To confirm PSCA protein we analyzed total cell lysates of tumor probes which have been rated positive or negative for PSCA expression (Fig. 2A). Immunoblot analysis demonstrated the appearance of PSCA protein expression in samples which were found to be PSCA-positive by QRT-PCR. Furthermore, when we tested five primary cell preparations established from freshly obtained glioblastoma tissue we detected PSCA expression in one GBM-preparation, designated HT7068, by FACS analysis and indirect immunofluorescence analysis using the monoclonal anti-PSCA antibody 7F5 (Fig. 2). FACS analysis on non-fixed cells and an indirect immunofluorescence analysis revealed the surface expression of PSCA. However, PSCA immunoreactivity ws also detected in the cytosol of the HT7068 glioblastoma cells (Fig. 2Bc and d).

PSCA expression levels are increased in malignant compared to benign brain tumors. PSCA-IR was mostly absent in
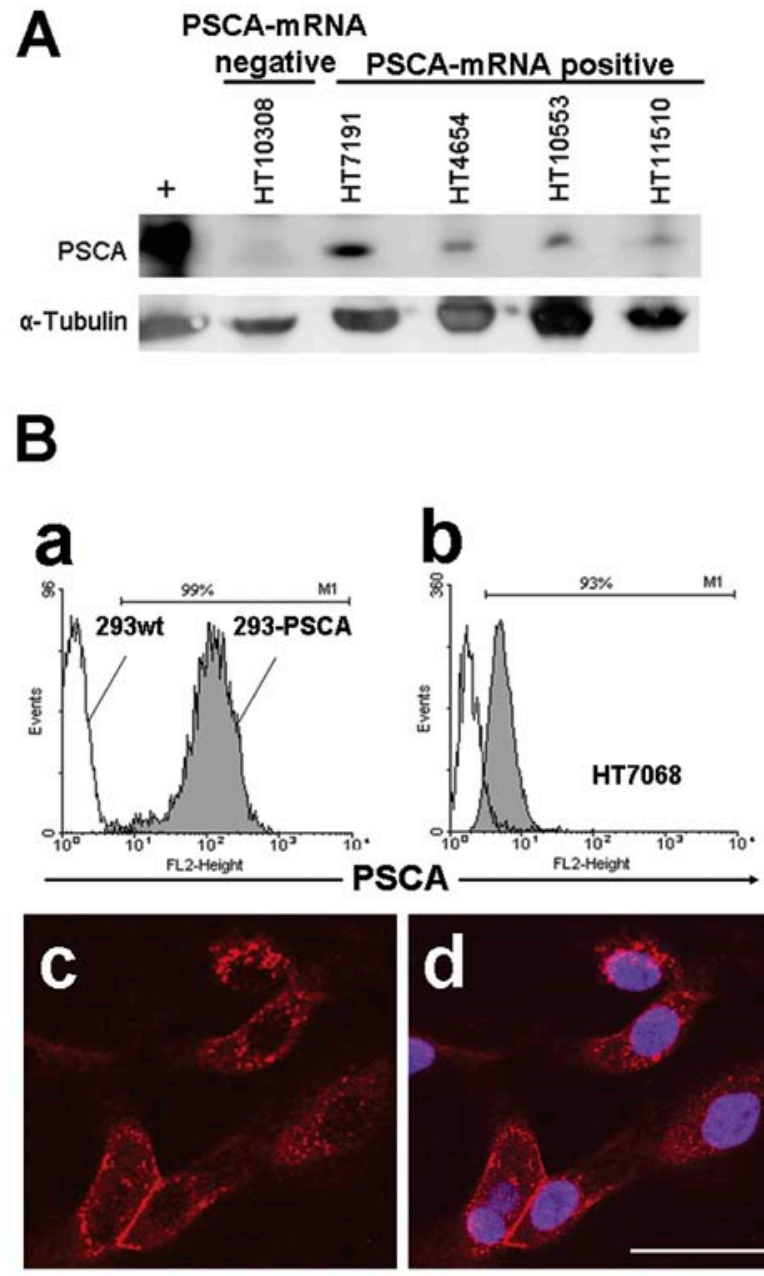

Figure 2. PSCA protein expression in glioblastoma probes and primary glioblastoma cells. (A) Total protein lysates from GBM tissues were subjected to $12 \%$ polyacrylamide gel electrophoresis. Blotted PSCA was detected with the monoclonal 7F5 PSCA antibody and secondary anti-mouse IgG coupled to HRP using ECL. As positive control (+) we included total protein lysate from $293 \mathrm{~T}$ cells transduced with a retroviral vector encoding PSCA (Material and methods). (B) Detection of PSCA protein in primary GBM cells. a, FACS analysis of PSCA expression in 293T cells with proviral expression of PSCA (grey histogram) using the monoclonal antibody 7F5 and secondary anti-mouse coupled to Cy3. As a control we included 293T wild-type cells (white histogram). b, FACS analysis showing PSCA expression in primary glioblastoma cells (HT7068) (grey histogram). An isotype control is included (white histogram). c, Confocal laser imaging of PSCA-immunoreactivity in HT7068 cells with the monoclonal 7F5 antibody and the secondary anti-mouse Cy3-labelled antibody. d, Additional nuclear counterstaining with Hoechst 33342. Note that PSCA is mostly localized to the cell membrane. Bar, $20 \mu \mathrm{m}$.

normal brain tissues. Specifically, reactive astrocytes in HIV-encephalopathy did not show PSCA-IR in any of the 10 analyzed cases (Figs. 3A and 4C). Only few astrocytes in hippocampal sclerosis of 2 cases showed minimal positive cytoplasmic staining (Figs. 3B and 4C). Of 28 benign WHO grade I and II tumors containing astrocytomas, ependymomas and oligodendrogliomas, we found varying PSCA-IR in the cell membranes, in the cytoplasm and in the cell processes of 12 cases (Figs. 3C and D, and 4C and D). The mean expression levels for PSCA in the different brain tumors of WHO grades I and II were very low with PSCA labeling scores of $\sim 0.5+$ (Fig. 4A). Only 2 astrocytomas of WHO grade II showed a PSCA score of 2+ (Figs. 3D and 4C). Since we found 

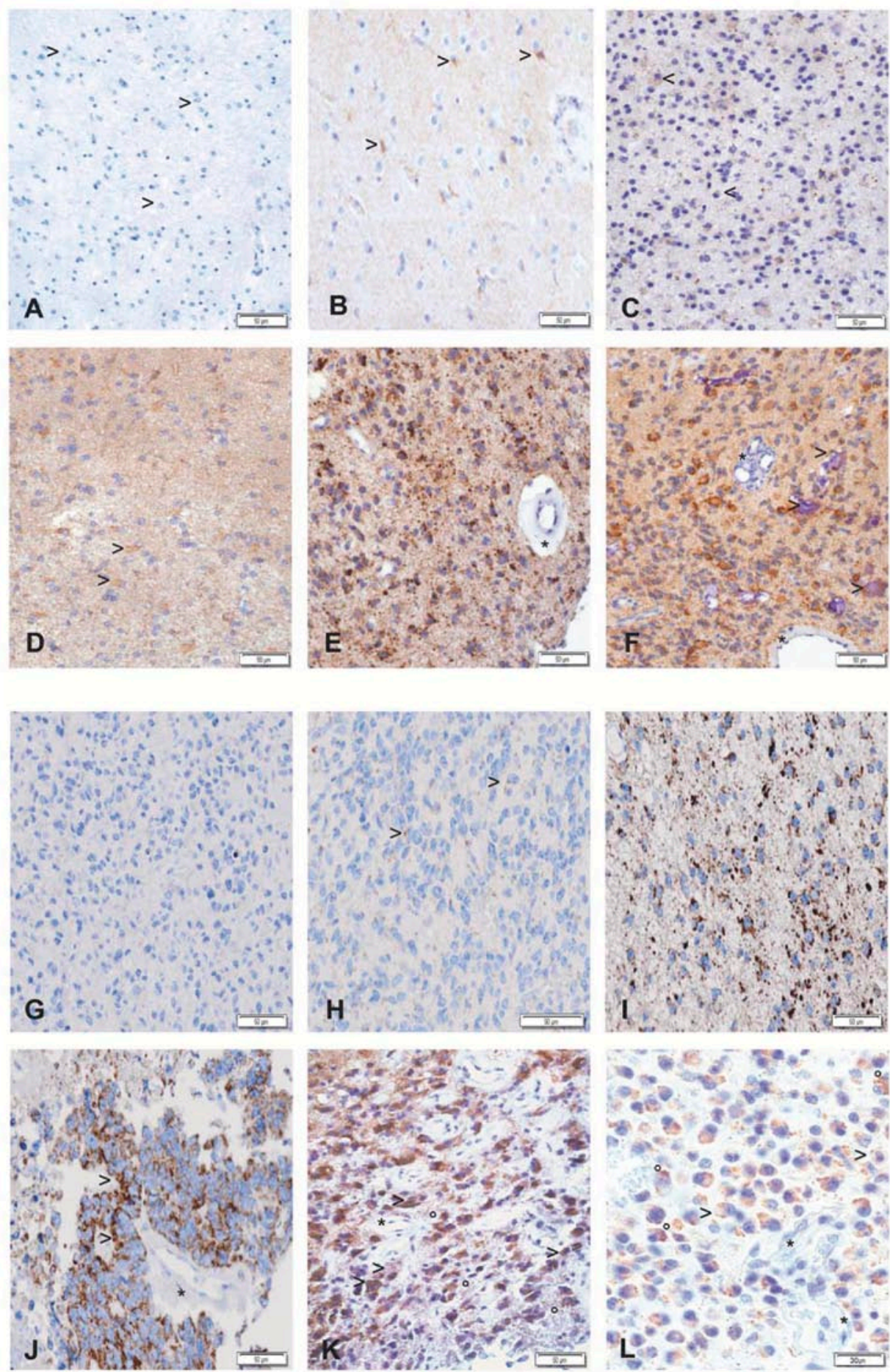

Figure 3. Immunohistochemical analyses of PSCA expression in reactive astrocytes and varying gliomas. (A-F) PSCA-IR in reactive astrocytes and in gliomas of varying types and dignities. (A) Reactive astrocytes in a patient with HIV-encephalitis (HIVe). Even clearly activated astrocytes with enlarged light nuclei and broad cytoplasm are negative for PSCA (arrowheads). (B) Astrogliosis in hippocampal sclerosis-induced epilepsy showing few astrocytes with low-level positive staining for PSCA (arrowheads). (C) Diffuse astrocytoma WHO II (astro II) with minimal PSCA-IR within glial processes (arrowheads). (D) Benign astrocytoma WHO II with diffuse increased PSCA-IR (score 2+) associated with glial processes and the cytoplasmic surface. (E) Anaplastic (malignant) astrocytoma WHO III (astro III) with strong expression of PSCA by IR on the cell surface and on disrupted plump glial processes leading to diffuse staining appearance. The vacular endothelium remains free of staining (asterisk). (F) Anaplastic (malignant) oligodendroglioma WHO III (oligo III) with similarly high PSCA-IR (score $3+)$ on the cell surface as in E denoting the lack of differentiation between different glioma types. The vascular endothelium (asterisk) and calcifications typical for oligodendroglioma (arrowheads) remain devoid of staining. (G-L) Representative images showing varying scores and patterns of PSCA-IR in 6 different cases of glioblastoma. PSCA expression was detected using the monoclonal 7F5 antibody and an indirect peroxidase method using DAB (brown) as a chromogen on sections of paraffin-embedded tissues as described in Materials and methods. (G) Glioblastoma completely negative for PSCA (score value, 0). (H) Glioblastoma with minimal PSCA-IR (score 1+, weak staining in $<50 \%$ of tumor cells) showing single astrocytic processes with positive IR and few cells with weak incomplete membranous IR (arrowheads). (I) Glioblastoma with moderate PSCA-IR (score 2+, positive staining of moderate intensity in 50-75\% of the tumor cells). (J) Glioblastoma with strong PSCA-IR (score $3+$, strong positive staining in $>75 \%$ with mostly complete membranous staining), vascular endothelium remains free of staining (asterisk). (K) Glioblastoma with strong PSCA-IR (score 3+) displaying a different staining pattern with mostly fibrillar staining and featuring frequently globular cytoplasmic staining (arrowheads) and prominent fibrillar staining of tumor cell processes (o). Blood vessels remain free of PSCA-expression (asterisk). (L) Gemistocytic glioblastoma with strong PSCA-IR (score 3+) displaying mostly membranous staining with some hyalin and globular cytoplasmic IR Again, blood vessels remain PSCA-negative (asterisk). Bars denote $50 \mu \mathrm{m}$ (original magnification, $\mathrm{x} 20$ ) for A-K; $30 \mu \mathrm{m}$ (original magnification, $\mathrm{x} 40$ ) for $\mathrm{L}$. 

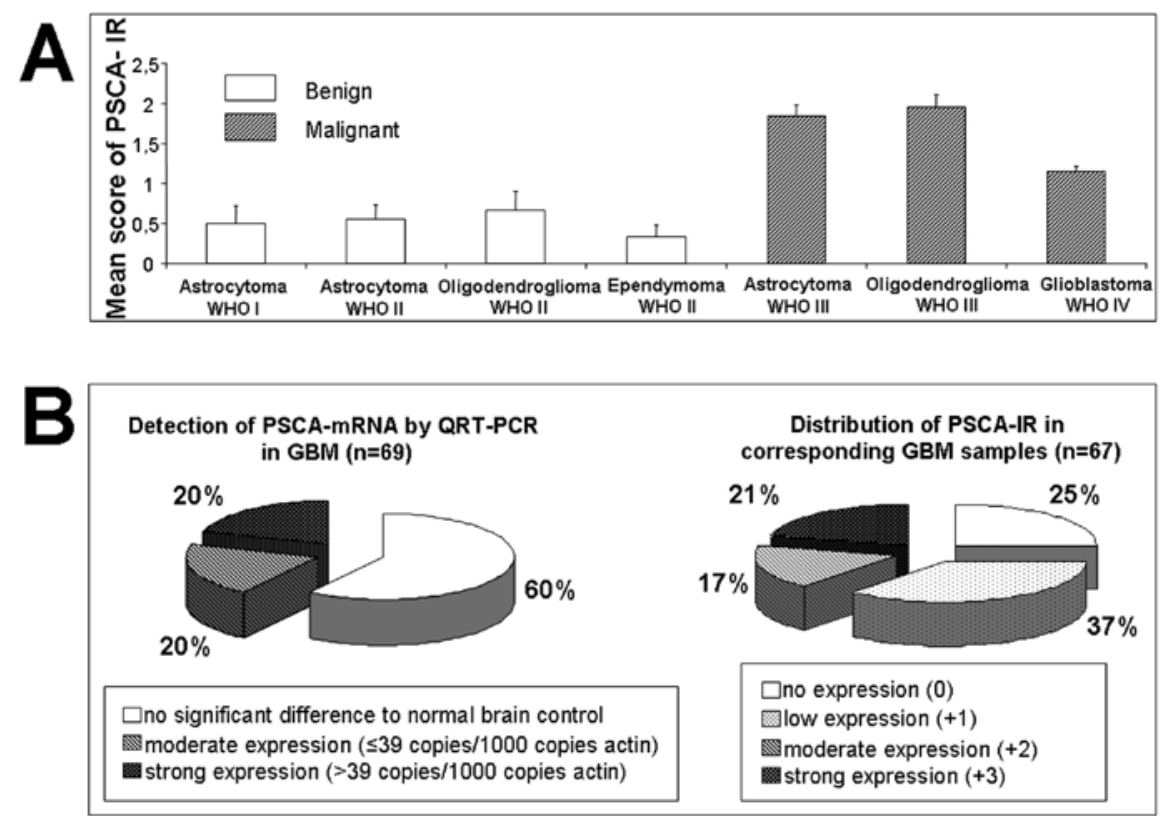

\begin{tabular}{|c|c|c|c|c|c|}
\hline \multirow[t]{2}{*}{ Tumor } & \multicolumn{4}{|c|}{ PSCA-scores } & \multirow[b]{2}{*}{ n } \\
\hline & 0 & +1 & +2 & +3 & \\
\hline Normal & 10 & 0 & 0 & 0 & 10 \\
\hline Epilepsy & 3 & 2 & 0 & 0 & 5 \\
\hline HIV-Encephalopathy & 10 & 0 & 0 & 0 & 10 \\
\hline Astrocytoma WHO I & 2 & 2 & 0 & 0 & 4 \\
\hline Astrocytoma WHO II & 7 & 3 & 2 & 0 & 12 \\
\hline Oligodendroglioma WHO II & 1 & 2 & 0 & 0 & 3 \\
\hline Ependymoma WHOII & 6 & 3 & 0 & 0 & 9 \\
\hline All WHO I-II & 16 & 10 & 2 & 0 & 28 \\
\hline Oligodendroglioma WHO III & 2 & 5 & 14 & 7 & 28 \\
\hline Astrocytoma WHO III & 0 & 5 & 13 & 2 & 20 \\
\hline Glioblastoma & 31 & 68 & 26 & 9 & 134 \\
\hline All WHO III-IV & 33 & 78 & 53 & 18 & 182 \\
\hline
\end{tabular}

\begin{tabular}{|c|c|c|c|c|}
\hline & & & $\begin{array}{c}p \\
\text { (chi-square) }\end{array}$ & $\begin{array}{l}\text { contingency } \\
\text { coeff.(100\%) }\end{array}$ \\
\hline \multicolumn{5}{|c|}{ 1. Correlation with malignancy of astrocytic tumors } \\
\hline Astro I - II & vs. & Astro III -IV & $<0.0001$ & $86 \%$ \\
\hline Astro III & vs. & Glioblastom IV & ns & \\
\hline \multicolumn{5}{|c|}{ 2. Differentiation between astrocytomas and oligodendrogliomas } \\
\hline Astro II & vs. & Oligo II & ns & \\
\hline Astro III & vs. & Oligo III & ns & \\
\hline Astro II & vs. & Epend II & ns & \\
\hline
\end{tabular}

Figure 4. PSCA expression in WHO grade I-II and WHO grade III-IV brain tumors and statistical evaluation. (A) PSCA-IR is associated with tumor malignancy but not with the type of glioma. Evaluation of PSCA expression levels in paraffin-embedded tissue sections. The graph represents the different types of glioma tested. Error bars denote the standard deviation of the means (SEM). (B) Comparison between PSCA mRNA and protein expression levels in GBM. The left graph shows the PSCA mRNA expression levels obtained by QRT-PCR, while the right graph depicts the distribution of different scores of IR on the same set of GBM samples. The distribution of PCR-results in the right graph is based on the expression scores described in Fig. 1. (C) Table showing the obtained PSCA-IR scores in the different brain tumor samples. Samples were evaluated as described in Materials and methods. (D) Statistical evaluation of PSCA-IR using the non-parametric $\chi^{2}$ test and the Pearson's contingency coefficient reveals that WHO grade III-IV gliomas significantly express more PSCA protein than astrocytomas of WHO grade II.

no statistical differences between WHO grade I and WHO grade II tumors, we grouped them together in order to obtain stronger statistical power regardless of potentially different origins of the different tumor entities when comparing them to WHO grade III-IV tumors (Fig. 4C).

In $\sim 40 \%$ of the glioblastoma samples studied by quantitative PCR ( $=69$ ), we found significant mRNA expression levels of PSCA and the corresponding PSCA protein (PSCA-IR scores $2+$ or $3+$ ) was detected in tissue blocks of $~ 38 \%$ of GBM samples ( $\mathrm{n}=67$; tissue blocks were not available for two patients) (Fig. 4B). However, for samples with low PSCA-IR score $(+1)$ we did not detect corresponding lower mRNA levels in the QRT-PCR analyses. Since we also observed intratumoral heterogeneity in PSCA-staining in these probes we increased the reliability of the study by enclosing further samples for immunohistochemistry to a total of 134 GBMs 
(Fig. 4A and C). In summary, 26\% of all tested GBM samples tested so far were found to have a PSCA labeling score of 2+ or 3+ (Fig. 4B). Again the distribution of PSCA-IR was not entirely homogeneous in these tested glioblastoma samples. The heterogeneity of all tested GBMs was reflected in the mean score value which was only slightly above 1+ (Fig. 4A). Increasing score values also indicate decreased heterogeneity. The value $3+$ denotes positive staining in $>75 \%$ of the tumor cells and the value of $2+$ was given for tumors having $>50 \%$ PSCA-positive cells. Cases with low-score values frequently showed heterogeneous staining results. PSCA signals were generally observed at the cell surfaces including astrocytic processes. Some cases with mainly gemistocytic appearance also displayed globular or diffuse cytoplasmic staining. Pointlike granular staining outside the cell body of gemistocytic tumors was mostly due to the irregular geometry of astrocytic processes (Fig. 3K and L). PSCA expression was not detected in the vascular walls and in the proliferating endothelium (Fig. 3E, F and J-L).

Interestingly, the majority of malignant glial tumors grade III and IV showed positive PSCA-IR of varying intensity (Figs. 3E-L and 4A). Noteworthy, we found a stronger and mostly uniform expression of PSCA protein in oligodendrogliomas $(n=28)$ and astrocytomas $(n=20)$ of WHO grade III when compared to GBM ( $\mathrm{n}=134)$ (Fig. 3E-L). Here, $75 \%$ of the analyzed tumors of WHO grade III showed PSCA staining scores of $2+$ and $3+$ resulting in a mean PSCA score of $\sim 2+$ for both tumor types (Fig. 4A and C). However, PSCA-IR did not discriminate between the WHO grades III and IV (Fig. 4A and D). Yet, the difference between the WHO grade I-II and WHO grade III-IV groups was statistically significant (Fig. 4D; $\mathrm{p}<0.001)$.

\section{Discussion}

The identification of new surface markers of brain tumors may be useful for diagnostic purposes and may also provide new target structures for antibody-guided therapies. In this study we demonstrated for the first time the presence of PSCA, a GPI-anchored surface protein in significant fractions of malignant glial tumors of WHO grades III and IV. In glioblastoma we recognized heterogeneous PSCA expression between individual tumor samples, but also an intratumoral heterogeneity. In part, these phenomena which have also been described for other markers, such as SOX2 and EGFR (13,31), may reflect the overall heterogeneity of GBMs. Although QRT-PCR data and immunohistochemistry data can not directly be compared there was a consistency in detecting tumors with moderate and high PSCA expression with both methods. For logistical reasons, we had to use different samples for QRT-PCR and immunohistochemistry, which might explain the slight differences at higher PSCA expression levels (Fig. 4B). Yet, there was an inconsistency in detection of GBMs with a PSCA protein labeling score of 1+ (low expression) and the detection with QRT-PCR, the latter showing no significant increased levels of PSCA mRNA when compared to normal brain tissue. This inconsistency might be due to the very accurate detection of single PSCA-positive cells when using the 7F5 monoclonal antibody and the semi-quantitative PSCA-IR scoring system which rates GBM specimens with only a few PSCA-positive cells or astrocytic processes with a PSCA score of 1+.

Interestingly, oligodendroglioma and astrocytoma of WHO grade III showed a strong and more uniform PSCA staining. Furthermore, it is remarkable that 12 out of 28 benign WHO grade I and II tumors were positive for PSCA, however with mostly lower levels of expression. This indicates that PSCA expression might be associated with tumor progression. It is noteworthy that a recent study provided evidence that some genetic variants of PSCA are associated with an increased risk of diffuse gastric cancer (32). It would be worthwhile to analyze the genetic variants of PSCA in high-grade glioma specimens and to investigate whether the expression of PSCA in low-grade tumors is associated with an increased risk of disease progression.

We did not find PSCA mRNA and protein expression in normal brain. In line with this, recent expression studies have shown that PSCA is predominantly prostate-specific (19), although low PSCA transcript levels and moderate protein levels have been detected in the transitional epithelium of the bladder and in the stomach $(28,29)$. PSCA expression is strongly up-regulated in prostate carcinoma (PCa) (19) and PCa metastases (20). In addition, PSCA was found to be strongly expressed in the majority of bladder carcinomas (21) and clear renal cell carcinoma (23). Therefore, PSCA seems to be exclusively expressed in cells and tumors of the urogenital system. However, other reports demonstrate the PSCA expression in cervical squamous cell carcinomas and endometrial carcinomas $(33,34)$. Furthermore, the recent discovery that pancreatic adenocarcinoma expressed PSCA (22) and our data describing PSCA expression in gliomas of WHO grades III and IV suggests a more universal role of PSCA in tumors of different origin. Yet, the function of PSCA in these tumors is not precisely known. Members of the Ly-6/Thy-1 gene family have been associated with increased malignant potential and metastasis (35). In line with this, a previous report demonstrated an attenuated tumor growth and activation of interferon $\alpha / \beta$-pathways in SW780 xenografts after conditional knock down of PSCA, suggesting that PSCA expression might have an inhibitory effect on the innate immune responses against tumors (36).

Interestingly, the avian ortholog of PSCA and the mouse PSCA were recently found to be expressed in the developing telencephalon and peripheral ganglia (37). Furthermore, they were suggested to play a role in antagonizing $\alpha 7$-containing nicotinic receptors and in preventing apoptosis during development. $\alpha 7$-containing nicotinic receptors have also been described for astrocytes and other non-excitable cells, vascular endothelial cells and keratinocytes (38). But so far no data concerning the role of PSCA in modulating apoptosis in astrocytic tumors, i.e. glioblastoma cells, is available.

Our immunohistochemical analyses of different gliomas of WHO grades III-IV revealed that PSCA was predominately expressed on the cell surface and sometimes found in the cytosol of the tumor cells. Therefore, for a fraction of patients PSCA might be a promising target structure for an antibodybased immunotherapy of gliomas, in particular when using $\mathrm{T}$ cells or NK cells endowed with a chimeric antigen receptor specific for PSCA. Recently, we have shown that a single chain antibody fragment derived form the monoclonal antibody 7F5 
used in this study can be utilized to construct a functional chimeric $\mathrm{T}$ cell receptor which confers $\mathrm{T}$ cells a selective cytotoxicity against PSCA-positive tumor cells (30).

Due to its absence from normal and most reactive astrocytes, PSCA may provide an additional tool to facilitate the identification of single tumor cells embedded in normal brain tissue, since frequently used markers such as the proliferation marker Ki67 (MIB-1) or TP53 do not allow for the definite recognition of single tumor cells. Likewise, in the future, PSCA may prove to be useful as a tool to determine the classification of brain tumors of borderline morphology or increased proliferation activity combined with bland morphology. However, further research will be necessary to elucidate the potential significance of PSCA as a prognostic marker in brain tumors.

In summary, we provide evidence, that PSCA is a novel marker for WHO grade III-IV gliomas. Therefore, for a number of patients with brain tumors, PSCA may represent an additional feasible target included in an immunotherapeutic approach targeting multiple glioma-associated antigens, in order to eliminate heterogeneous glioma cell populations within the tumor.

\section{Acknowledgements}

This study was supported by a grant from the Deutsche Krebshilfe e.V. (grant no. 108009) to A.T. We thank B. Hamann, B. Liebscher, B. Peterson, M. Krenz and F. Zachow for excellent technical assistance.

\section{References}

1. Louis DN, Ohgaki H, Wiestler OD, Cavenee WK, Burger PC, Jouvet A, Scheithauer BW and Kleihues P: The 2007 WHO classification of tumours of the central nervous system. Acta Neuropathol 114: 97-109, 2007.

2. Kocher M, Frommolt P, Borberg SK, Ruhl U, Steingraber M, Niewald M, Staar S, Stuschke M, Becker G, Fischedick AR, Herfarth K, Grauthoff $\mathrm{H}$ and Muller RP: Randomized study of postoperative radiotherapy and simultaneous temozolomide without adjuvant chemotherapy for glioblastoma. Strahlenther Onkol 184: 572-579, 2008.

3. Sampson JH, Archer GE, Ashley DM, Fuchs HE, Hale LP, Dranoff $G$ and Bigner DD: Subcutaneous vaccination with irradiated, cytokine-producing tumor cells stimulates $\mathrm{CD} 8^{+}$ cell-mediated immunity against tumors located in the 'immunologically privileged' central nervous system. Proc Natl Acad Sci USA 93: 10399-10404, 1996.

4. Okada H, Kohanbash G, Zhu X, Kastenhuber ER, Hoji A, Ueda R and Fujita M: Immunotherapeutic approaches for glioma. Crit Rev Immunol 29: 1-42, 2009.

5. Yu JS, Wheeler CJ, Zeltzer PM, Ying H, Finger DN, Lee PK, Yong WH, Incardona F, Thompson RC, Riedinger MS, Zhang W, Prins RM and Black KL: Vaccination of malignant glioma patients with peptide-pulsed dendritic cells elicits systemic cytotoxicity and intracranial T-cell infiltration. Cancer Res 61: 842-847, 2001.

6. Ueda R, Kinoshita E, Ito R, Kawase T, Kawakami Y and Toda M Induction of protective and therapeutic antitumor immunity by a DNA vaccine with a glioma antigen, SOX6. Int J Cancer 122: 2274-2279, 2008.

7. Kuan CT, Wikstrand CJ and Bigner DD: EGFRvIII as a promising target for antibody-based brain tumor therapy. Brain Tumor Pathol 17: 71-78, 2000.

8. Ahmed N, Ratnayake M, Savoldo B, Perlaky L, Dotti G, Wels WS, Bhattacharjee MB, Gilbertson RJ, Shine HD, Weiss HL, Rooney CM, Heslop HE and Gottschalk S: Regression of experimental medulloblastoma following transfer of HER2-specific T cells. Cancer Res 67: 5957-5964, 2007.
9. Liu G, Ying H, Zeng G, Wheeler CJ, Black KL and Yu JS: HER-2, gp100, and MAGE-1 are expressed in human glioblastoma and recognized by cytotoxic T cells. Cancer Res 64: 4980-4986, 2004.

10. Zhang JG, Eguchi J, Kruse CA, Gomez GG, Fakhrai H, Schroter S, Ma W, Hoa N, Minev B, Delgado C, Wepsic HT, Okada H and Jadus MR: Antigenic profiling of glioma cells to generate allogeneic vaccines or dendritic cell-based therapeutics. Clin Cancer Res 13: 566-575, 2007

11. Komata T, Kanzawa T, Kondo Y and Kondo S: Telomerase as a therapeutic target for malignant gliomas. Oncogene 21: 656-663, 2002.

12. Katoh M, Wilmotte R, Belkouch MC, De Tribolet N, Pizzolato G and Dietrich PY: Survivin in brain tumors: an attractive target for immunotherapy. J Neurooncol 64: 71-76, 2003.

13. Schmitz M, Temme A, Senner V, Ebner R, Schwind S, Stevanovic S, Wehner R, Schackert G, Schackert HK, Fussel M, Bachmann M, Rieber EP and Weigle B: Identification of SOX2 as a novel glioma-associated antigen and potential target for $\mathrm{T}$ cell-based immunotherapy. Br J Cancer 96: 1293-1301, 2007.

14. Schmitz M, Wehner R, Stevanovic S, Kiessling A, Rieger MA, Temme A, Bachmann M, Rieber EP and Weigle B: Identification of a naturally processed $\mathrm{T}$ cell epitope derived from the gliomaassociated protein SOX11. Cancer Lett 245: 331-336, 2007.

15. Weigle B, Ebner R, Temme A, Schwind S, Schmitz M, Kiessling A, Rieger MA, Schackert G, Schackert HK and Rieber EP: Highly specific overexpression of the transcription factor SOX11 in human malignant gliomas. Oncol Rep 13: 139-144, 2005.

16. Ehtesham M, Black KL and Yu JS: Recent progress in immunotherapy for malignant glioma: treatment strategies and results from clinical trials. Cancer Control 11: 192-207, 2004.

17. Antica M, Wu L and Scollay R: Stem cell antigen 2 expression in adult and developing mice. Immunol Lett 55: 47-51, 1997.

18. Classon BJ and Coverdale L: Mouse stem cell antigen Sca-2 is a member of the Ly- 6 family of cell surface proteins. Proc Natl Acad Sci USA 91: 5296-5300, 1994.

19. Reiter RE, Gu Z, Watabe T, Thomas G, Szigeti K, Davis E, Wahl M, Nisitani S, Yamashiro J, Le Beau MM, Loda M and Witte ON: Prostate stem cell antigen: a cell surface marker overexpressed in prostate cancer. Proc Natl Acad Sci USA 95: 1735-1740, 1998.

20. Lam JS, Yamashiro J, Shintaku IP, Vessella RL, Jenkins RB, Horvath S, Said JW and Reiter RE: Prostate stem cell antigen is overexpressed in prostate cancer metastases. Clin Cancer Res 11: 2591-2596, 2005.

21. Amara N, Palapattu GS, Schrage M, Gu Z, Thomas GV, Dorey F, Said $\mathrm{J}$ and Reiter RE: Prostate stem cell antigen is overexpressed in human transitional cell carcinoma. Cancer Res 61: 4660-4665, 2001.

22. Argani P, Rosty C, Reiter RE, Wilentz RE, Murugesan SR, Leach SD, Ryu B, Skinner HG, Goggins M, Jaffee EM, Yeo CJ, Cameron JL, Kern SE and Hruban RH: Discovery of new markers of cancer through serial analysis of gene expression: prostate stem cell antigen is overexpressed in pancreatic adenocarcinoma. Cancer Res 61: 4320-4324, 2001.

23. Elsamman EM, Fukumori T, Tanimoto S, Nakanishi R, Takahashi M, Toida K and Kanayama HO: The expression of prostate stem cell antigen in human clear cell renal cell carcinoma: a quantitative reverse transcriptase-polymerase chain reaction analysis. BJU Int 98: 668-673, 2006.

24. Tanaka M, Komatsu N, Terakawa N, Yanagimoto Y, Oka M, Sasada T, Mine T, Gouhara S, Shichijo S, Okuda S and Itoh K: Increased levels of IgG antibodies against peptides of the prostate stem cell antigen in the plasma of pancreatic cancer patients. Oncol Rep 18: 161-166, 2007.

25. Kiessling A, Schmitz M, Stevanovic S, Weigle B, Holig K, Fussel M, Fussel S, Meye A, Wirth MP and Rieber EP: Prostate stem cell antigen: Identification of immunogenic peptides and assessment of reactive $\mathrm{CD} 8^{+} \mathrm{T}$ cells in prostate cancer patients. Int J Cancer 102: 390-397, 2002.

26. Garcia-Hernandez ML, Gray A, Hubby B, Klinger OJ and Kast M: Prostate stem cell antigen vaccination induces a long-term protective immune response against prostate cancer in the absence of autoimmunity. Cancer Res 68: 861-869, 2008.

27. Saffran DC, Raitano AB, Hubert RS, Witte ON, Reiter RE and Jakobovits A: Anti-PSCA mAbs inhibit tumor growth and metastasis formation and prolong the survival of mice bearing human prostate cancer xenografts. Proc Natl Acad Sci USA 98: 2658-2663, 2001. 
28. Ross S, Spencer SD, Holcomb I, Tan C, Hongo J, Devaux B, Rangell L, Keller GA, Schow P, Steeves RM, Lutz RJ, Frantz G, Hillan K, Peale F, Tobin P, Eberhard D, Rubin MA, Lasky LA and Koeppen H: Prostate stem cell antigen as therapy target: tissue expression and in vivo efficacy of an immunoconjugate. Cancer Res 62: 2546-2553, 2002.

29. Cunha AC, Weigle B, Kiessling A, Bachmann M and Rieber EP: Tissue-specificity of prostate specific antigens: comparative analysis of transcript levels in prostate and non-prostatic tissues. Cancer Lett 236: 229-238, 2006.

30. Morgenroth A, Cartellieri M, Schmitz M, Gunes S, Weigle B, Bachmann M, Abken H, Rieber EP and Temme A: Targeting of tumor cells expressing the prostate stem cell antigen (PSCA) using genetically engineered T-cells. Prostate 67: 1121-1131, 2007.

31. Strommer K, Hamou MF, Diggelmann H and de TN: Cellular and tumoural heterogeneity of EGFR gene amplification in human malignant gliomas. Acta Neurochir (Wien) 107: 82-87, 1990.

32. Lu Y, Chen J, Ding Y, Jin G, Wu J, Huang H, Deng B, Hua Z, Zhou Y, Shu Y, Liu P, Hu Z, Shen J, Xu Y and Shen H: Genetic variation of PSCA gene is associated with the risk of both diffuse-and intestinal-type gastric cancer in a Chinese population. Int J Cancer 127: 2183-2189, 2010.
33. Liu WK, Jiang XY and Zhang ZX: Expression of PSCA, PIWIL1 and TBX2 and its correlation with HPV16 infection in formalinfixed, paraffin-embedded cervical squamous cell carcinoma specimens. Arch Virol 155: 657-663, 2010.

34. Liu WK, Jiang XY and Zhang ZX: Expression of PSCA, PIWIL1, and TBX2 in endometrial adenocarcinoma. Onkologie 33: 241-245, 2010.

35. Witz IP: Differential expression of genes by tumor cells of a low or a high malignancy phenotype: the case of murine and human Ly-6 proteins. J Cell Biochem (Suppl) 34: 61-66, 2000.

36. Marra E, Uva P, Viti V, Simonelli V, Dogliotti E, De RE, Lahm A, La MN, Nicosia A, Ciliberto G and Palombo F: Growth delay of human bladder cancer cells by prostate stem cell antigen downregulation is associated with activation of immune signaling pathways. BMC Cancer 10: 129, 2010.

37. Hruska M, Keefe J, Wert D, Tekinay AB, Hulce JJ, Ibanez-Tallon I and Nishi R: Prostate stem cell antigen is an endogenous lynx1-like prototoxin that antagonizes alpha7-containing nicotinic receptors and prevents programmed cell death of parasympathetic neurons. J Neurosci 29: 14847-14854, 2009.

38. Sharma G and Vijayaraghavan S: Nicotinic receptor signaling in nonexcitable cells. J Neurobiol 53: 524-534, 2002. 